\title{
Drug duo increases therapeutic window
}

They're a mainstay of anti-inflammatory agents, yet there's no denying glucocorticoids have undesirable side effects. Efforts to increase the therapeutic window of glucocorticoids mainly focus on medicinal chemistry strategies, but a new systems-biology approach now shows that the anti-inflammatory activity of very-low-dose glucocorticoid can be amplified without increasing associated adverse effects. Grant Zimmermann et al. have successfully used the anti-thrombotic drug dipyridamole to enhance the antiinflammatory activity of prednisolone, as published in Arthritis Research \& Therapy.

First, the authors showed that dipyridamole and prednisolone synergistically suppressed the secretion of tumor necrosis factor (TNF) from human peripheral blood mononuclear cells stimulated with lipopolysaccharide in vitro, meaning that a lower dose of these agents could be used. Next, they tested these agents in vivo. In acute inflammation models, a combination of these agents reduced TNF production at doses suggestive of an up to 10 -fold amplification of prednisolone-mediated anti-inflammatory activity by dipyridamole. In a rat model of collagen-induced arthritis, the combination of prednisolone and dipyridamole at $0.3 \mathrm{mg} / \mathrm{kg}$ and $150 \mathrm{mg} / \mathrm{kg}$, respectively, reduced swelling in the joints; on its own, $3 \mathrm{mg} / \mathrm{kg}$ prednisolone was required to invoke a similar reduction in swelling. The combination treatment reduced the average arthritis score to below that achieved by low-dose prednisolone or dipyridamole alone, and further radiological and histological analysis indicated that dipyridamole could amplify

\section{Combination drugs may} achieve greater levels of activity, and higher degrees of selectivity... 77

the activity of prednisolone in reducing joint space narrowing, the presence of erosions, cartilage and bone damage, inflammation and pannus formation.

Notably, the amplifying effects of dipyridamole on prednisolone were restricted to its anti-inflammatory effects rather than any other adverse effects, such that the expression levels of tyrosine aminotransferase in the liver (as a marker of glucocorticoid-activated glucose metabolism) were comparable in animals treated with low-dose prednisolone and with both low-dose prednisolone and dipyridamole. Dipyridamole did not amplify any effect of low-dose prednisolone on the suppression of corticosterone, nor did it affect adrenal gland or thymus weights, or the prednisolone-induced reduction of osteocalcin and mid-femur bone density. In corticotrophs, as an example of a different cellular context, dipyridamole failed to amplify the suppressive effect of prednisolone on pro-opiomelanocortin expression or adrenocorticotropic hormone secretion.

The researchers have demonstrated the success of a multi-target approach in increasing the therapeutic window of prednisolone, which Zimmermann believes "may represent a general method for achieving cell-type-specific amplification and dissociation of the pharmacological activity of nuclear hormone receptor ligands". An enhancing agent-in this case, dipyridamole'sensitizes' immune cells to very low doses of prednisolone by modulating specific signaling pathways that can amplify the anti-inflammatory effect. The cellular networks of other cells, such as corticotrophs, don't support such amplification, so low doses of prednisolone, in combination with dipyridamole, have the desired anti-inflammatory effect with a satisfactory safety profile at the wholeorganism level. "Combination drugs may achieve greater levels of activity, and higher degrees of selectivity, than can be achieved by highly target-selective single agent drugs," according to Zimmermann.

\section{Katrin Legg}

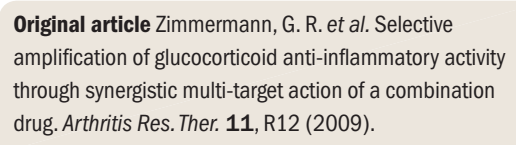

